\title{
QCD Rescattering and High Energy Two-Body Photodisintegration of the Deuteron
}

\author{
Leonid L. Frankfurt, ${ }^{1}$ Gerald A. Miller, ${ }^{2}$ Misak M. Sargsian, ${ }^{3, *}$ and Mark I. Strikman ${ }^{4}$ \\ ${ }^{1}$ School of Physics and Astronomy, Tel Aviv University, Tel Aviv 79978, Israel \\ ${ }^{2}$ Department of Physics, University of Washington, Box 351560, Seattle, Washington 98195-1560 \\ ${ }^{3}$ Department of Physics, Florida International University, Miami, Florida 33199 \\ ${ }^{4}$ Department of Physics, Pennsylvania State University, University Park, Pennsylvania 16802
}

(Received 9 April 1999)

\begin{abstract}
Photon absorption by a quark in one nucleon followed by its high-momentum transfer interaction with a quark in the other may produce two final-state nucleons with high relative momentum. We sum the relevant quark rescattering diagrams to show that the scattering amplitude depends on a convolution between the large angle $p n$ scattering amplitude, the hard photon-quark interaction vertex, and the lowmomentum deuteron wave function. The computed absolute values of the cross section are in reasonable agreement with the data.
\end{abstract}

PACS numbers: 24.85.+p, 12.38.Qk, 25.10.+s, 25.20.-x

The recent experiments on high energy two-body photodisintegration of the deuteron [1-3] set a new stage in high energy $\left(E_{\gamma} \geq 1 \mathrm{GeV}\right)$ nuclear physics. The calculations using the conventional mesonic picture of nuclear interactions failed to describe the qualitative features of these measurements. Thus these experiments are unique in testing the implications of quantum chromodynamics (QCD) in nuclear reactions $[4,5]$.

The hypothesis that the Fock state with the smallest number of partonic constituents dominates in the two-body large angle hard collisions predicts with considerable success the scaling behavior of the fixed angle differential cross sections [6]. In particular, the cross section of the fixed angle $\gamma d \rightarrow p n$ reaction should scale as $d \sigma / d t \sim$ $s^{-11}$. This scaling idea is successful in describing a number of processes, but it leads to many unanswered questions $[7,8]$. In particular, what suppresses the contributions of components of the hadronic wave function consisting of a single fast parton and wee spectator partons (Feynman mechanism) [9]? In a $p \mathrm{QCD}$ analysis of exclusive reactions, one is faced with the additional problem of describing the absolute value of the cross section [in reactions involving baryons, the calculations underestimate measured cross sections by orders of magnitude (see, e.g., [7])]. Examining the data [1] shows that the situation is rather complicated for $\gamma d \rightarrow p n$ reactions too. Scaling is observed at $\theta_{\text {c.m. }} \approx 89^{\circ}, 69^{\circ}$, but not for smaller angles. No existing model is able to calculate the absolute value of the cross section [1].

Here we investigate the mechanism in which the absorption of the photon by a quark of one nucleon, followed by a high-momentum transfer (hard) rescattering with a quark from the second nucleon, produces the final two nucleon state of large relative momentum. We calculate the contribution due to the low momentum $(<300 \mathrm{MeV} / c)$ of nucleons in the deuteron. This contribution is dominant since the deuteron is a loosely bound system. Another contribution [10], occurring when the photon breaks up a preexisting high relative momentum state in the deuteron, depends on the deuteron wave function evaluated at relative nucleon momenta $\sim$ few $\mathrm{GeV} / c$.

The validity of our hard rescattering mechanism requires several kinematic conditions. The use of the partonic picture requires that the masses of the intermediate hadronic state produced by the $\gamma N$ interaction be larger than some minimum mass characterizing the threshold to reach the continuum. This is known from deep inelastic scattering [9] to be $W \approx 2.2 \mathrm{GeV}$. Here the mass of the intermediate (between the photon absorption and quark rescattering) virtual state is $m_{\text {int }} \sim \sqrt{2 E_{\gamma} m_{N}}$. From the condition $m_{\text {int }} \geq W$ one obtains $E_{\gamma} \geq 2.5 \mathrm{GeV}$. Next, the struck quark (Fig. 1) should be energetic enough to reach the quark of the other nucleon without radiating soft (bremsstrahlung) gluons. Such radiation is characterized by a regeneration distance $l_{r} \approx E_{\gamma} R^{2}$ [11], where $R \approx 0.3 \mathrm{fm}$ characterizes the confinement radius. For $E_{\gamma} \geq 2.5$ we obtain $l_{r} \geq 1.1 \mathrm{fm}$ is larger than the nucleon radius and comparable with relevant internucleon distances in deuteron. Finally, to ensure that the quark rescattering is hard enough, one requires that the transverse momentum of final nucleons $p_{t} \geq 1 \mathrm{GeV}$ which requires $-t,-u \gtrsim 2 \mathrm{GeV}^{2}$.

Our derivation proceeds by evaluating Feynman diagrams such as Fig. 1. The quark interchange mechanism (see, e.g., [12]), in which quarks are exchanged between nucleons via the exchange of a gluon and the recoil quark-gluon system is on mass shell, is used. All other quark interactions are included in the nonperturbative partonic wave function of the nucleon, $\psi_{N}$.

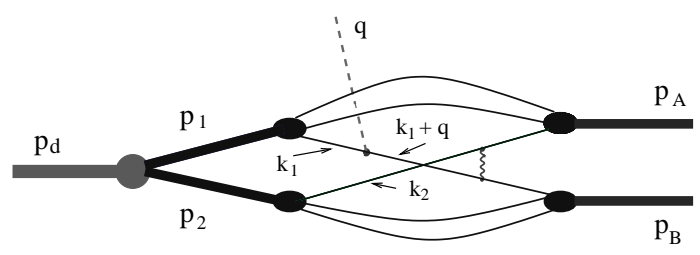

FIG. 1. Quark rescattering diagram. 
One proceeds (see, e.g., $[13,14]$ ) by integrating over the minus momenta [15] to obtain an expression involving only three-dimensional integrals. The result is that the deuteron- $N N$ vertices are replaced by the light-cone deuteron wave function, $\Psi_{d}$, and nucleon-(quark,gluon) vertices are replaced by $\psi_{N}$. We use a simplified notation in which only the momenta of the interacting quarks are labeled. Thus the scattering amplitude $T$ for photodisintegration of a deuteron $\left(p_{d}\right.$, mass $\left.M_{d}\right)$ into two nucleons $\left(p_{A}\right.$ and $\left.p_{B}\right)$ is

$$
\begin{aligned}
T=- & \sum_{e_{q}} \int\left(\frac{\psi_{N}^{\dagger}\left(x_{2}^{\prime}, p_{B \perp}, k_{2 \perp}\right)}{x_{2}^{\prime}} \bar{u}\left(p_{B}-p_{2}+k_{2}\right)\left[-i g T_{c}^{F} \gamma^{\nu}\right] \frac{u\left(k_{1}+q\right) \bar{u}\left(k_{1}+q\right)}{\left(k_{1}+q\right)^{2}-m_{q}^{2}+i \epsilon}\left[-i e_{q} \epsilon^{\perp} \gamma^{\perp}\right] u\left(k_{1}\right)\right. \\
& \left.\times \frac{\psi_{N}\left(x_{1}, p_{1 \perp}, k_{1 \perp}\right)}{x_{1}}\right)\left\{\frac{\psi_{N}^{\dagger}\left(x_{1}^{\prime}, p_{A \perp}, k_{1 \perp}\right)}{x_{1}^{\prime}} \bar{u}\left(p_{A}-p_{1}+k_{1}\right)\left[-i g T_{c}^{F} \gamma_{\mu}\right] u\left(k_{2}\right) \frac{\psi_{N}\left(x_{2}, p_{2 \perp}, k_{2 \perp}\right)}{x_{2}}\right\} \\
& \times G^{\mu \nu} \frac{\Psi_{d}\left(\alpha, p_{\perp}\right)}{1-\alpha} \frac{d x_{1}}{1-x_{1}} \frac{d^{2} k_{1 \perp}}{2(2 \pi)^{3}} \frac{d x_{2}}{1-x_{2}} \frac{d^{2} k_{2 \perp}}{2(2 \pi)^{3}} \frac{d \alpha}{\alpha} \frac{d^{2} p_{\perp}}{2(2 \pi)^{3}} .
\end{aligned}
$$

The deuteron is composed of nucleons of momenta $p_{1} \quad$ and $\quad p_{2} \quad$ with $\alpha \equiv \frac{p_{1+}}{p_{d+}}, \quad p_{2}=p_{d}-p_{1}$, and $p_{1 \perp}=-p_{2 \perp} \equiv p_{\perp}$ [15]. Each of these consists of one active quark of momenta $k_{1}$ and $k_{2}$ and a residual quark-gluon spectator system of momenta $p_{1}-k_{1}$ and $p_{2}-k_{2}$. It is useful to define the momentum fractions: $\quad x_{1} \equiv \frac{k_{1+}}{p_{1+}}=\frac{k_{1+}}{\alpha p_{d+}}, \quad x_{2} \equiv \frac{k_{2+}}{p_{2+}}=\frac{k_{2+}}{(1-\alpha) p_{d+}}$, $1-x_{1}^{\prime}=\frac{p_{1+}-k_{1+}}{p_{F+}}$, and $1-x_{2}^{\prime} \equiv \frac{p_{2+}-k_{2+}}{p_{B+}}$. The amplitude in Eq. (1) is a convolution of several blocks. (a) $\Psi_{d}\left(\alpha, p_{2 \perp}\right)$ describes the transition of the deuteron into a two-nucleon system. (b) The term in the (..) describes the "knocking out" of the quark of one nucleon by the incoming photon, with intact quark-gluon recoil of interacting nucleon and subsequent gluon exchange of that quark with the quark of the second nucleon. It consists of $\psi_{N}$; the $\gamma$-quark vertex $-i e_{q} \epsilon^{\perp} \cdot \gamma^{\perp}$, where $\epsilon^{\perp}$ is the polarization vector of the incoming photon; the intermediate-state propagator of the knocked-out quark $\frac{u \bar{u}}{\left(k_{1}+q\right)^{2}-m_{q}^{2}+i \epsilon}$ [16], with current quark mass $m_{q}$; the quark-gluon vertex $=i g T_{c}^{F} \gamma^{\mu}$; and the wave function of the final nucleon $\psi_{N}^{\dagger}$. (c) The expression in $\{\cdots\}$ describes the interaction of the quark from the second nucleon with the knocked-out quark. (d) The propagator of the exchanged gluon is $G^{\mu \nu}=\frac{i d_{\mu \nu}}{\left[l-q+\left(p_{1}-k_{1}\right)-\left(p_{2}-k_{2}\right)\right]^{2}+i \epsilon}$ with polarization matrix $d_{\mu \nu}$ (fixed using the light-cone gauge) and $l \equiv\left(p_{B}-p_{1}\right)$. We use the reference frame where $p_{d}=\left(p_{d 0}, p_{d z}, p_{\perp}\right) \equiv\left[\left(\sqrt{s^{\prime}} / 2\right)+\left(M_{d}^{2} / 2 \sqrt{s^{\prime}}\right),\left(\sqrt{s^{\prime}} / 2\right)-\right.$ $\left.\left(M_{d}^{2} / 2 \sqrt{s^{\prime}}\right), 0\right]$, with $s=\left(q+p_{d}\right)^{2}, s^{\prime} \equiv s-M_{d}^{2}$, and $q^{\mu}=\left[\left(\sqrt{s^{\prime}} / 2\right),-\left(\sqrt{s^{\prime}} / 2\right), 0_{\perp}\right]$.
To proceed we analyze the denominator of the knocked-out quark propagator when the recoil quark-gluon system is on-mass shell. We are concerned with momenta such that $p_{\perp}^{2} \ll m_{N}^{2} \ll s^{\prime}$ and $\alpha \sim \frac{1}{2}$ so we neglect terms of order $p_{\perp}^{2}, m_{N}^{2} / s^{\prime} \ll 1$ to obtain

$$
\left(k_{1}+q\right)^{2}-m_{q}^{2}+i \epsilon \approx x_{1} s^{\prime}\left(\alpha-\alpha_{c}+i \epsilon\right),
$$

where $\alpha_{c} \equiv\left[\left(x_{1} m_{R}^{2}+k_{1 \perp}^{2}\right) /\left(1-x_{1}\right) x_{1} \tilde{s}\right], \quad \tilde{s} \equiv s^{\prime}[1+$ $\left.\left(M_{d}^{2} / s^{\prime}\right)\right]$, and $m_{R}$ is the recoil mass of the spectator quark-gluon system of the first nucleon. The deuteron wave function is very strongly peaked at $\alpha=\frac{1}{2}$ and $p_{\perp}=0$, so the dominant contribution to $T$ corresponds to $\alpha_{c} \approx \frac{1}{2}$. According to Eq. (2) the integration in Eq. (1) over $k_{1 \perp}$ in the region $k_{1 \perp}^{2} \sim \frac{\left(1-x_{1}\right) x_{1} \tilde{s}}{2} \gg x_{1} m_{R}^{2}$ does provide $\alpha_{c}=\frac{1}{2}$. Keeping only the imaginary part of the quark propagator (the knocked-out quark is on-mass shell in the intermediate state) leads to $\alpha=\alpha_{c}$ and corresponds to keeping the contribution from the soft component of the deuteron wave function. Another consequence of on-mass shellness of the knock-out quark (and recoil quark-gluon system) is that gauge invariance and conservation of electromagnetic current are easily fulfilled.

Next we calculate the photon-quark hard scattering vertex, $\bar{u}\left(k_{1}+q\right)\left[\gamma_{\perp}\right] u\left(k_{1}\right)$, and use Eq. (2) to integrate over $\alpha$, by taking into account only the second term in the decomposition of the struck quark propagator: $\left(\alpha-\alpha_{c}+\right.$ $\epsilon)^{-1} \equiv \mathcal{P}\left(\alpha-\alpha_{c}\right)^{-1}-i \pi \delta\left(\alpha-\alpha_{c}\right)$ :

$$
\begin{aligned}
T= & \frac{i}{2} \sum_{q} \frac{e_{q}\left(\epsilon^{+}+\epsilon^{-}\right)}{2 \sqrt{s^{\prime}}} \int\left[\left(\frac{\left(1-\alpha_{c} x_{1}\right)^{2}+\frac{4 k_{1 \perp}^{2}}{s^{\prime}}}{\alpha_{c}^{2} x_{1}^{2}+\frac{4 k_{1 \perp}^{2}}{s^{\prime}}}\right)^{1 / 4} \alpha_{c} x_{1}+\left(\frac{\alpha_{c}^{2} x_{1}^{2}+\frac{4 k_{1 \perp}^{2}}{s^{\prime}}}{\left(1-\alpha_{c} x_{1}\right)^{2}+\frac{4 k_{1 \perp}^{2}}{s^{\prime}}}\right)^{1 / 4}\left(1-\alpha_{c} x_{1}\right)\right] \\
& \times \frac{1}{x_{1}} \frac{\psi_{N}^{\dagger}\left(x_{2}^{\prime}, p_{B \perp}, k_{2 \perp}\right)}{x_{2}^{\prime}} \bar{u}\left(p_{B}-p_{2}+k_{2}\right)\left[-i g T_{c}^{F} \gamma^{\nu}\right] u\left(k_{1}+q\right) \frac{\psi_{N}\left(x_{1}, p_{1 \perp}, k_{1 \perp}\right)}{x_{1}} \frac{\psi_{N}^{\dagger}\left(x_{1}^{\prime}, p_{F \perp}, k_{1 \perp}\right)}{x_{1}^{\prime}} \\
& \times \bar{u}\left(p_{A}-p_{1}+k_{1}\right)\left[-i g T_{c}^{F} \gamma_{\mu}\right] u\left(k_{2}\right) \frac{\psi_{N}\left(x_{2}, p_{2 \perp}, k_{2 \perp}\right)}{x_{2}} G^{\mu \nu} \frac{d x_{1}}{1-x_{1}} \frac{d^{2} k_{1 \perp}}{2(2 \pi)^{3}} \frac{d x_{2}}{1-x_{2}} \frac{d^{2} k_{2 \perp}}{2(2 \pi)^{3}} \frac{\Psi_{d}\left(\alpha_{c}, p_{\perp}\right)}{\alpha_{c}\left(1-\alpha_{c}\right)} \frac{d^{2} p_{\perp}}{2(2 \pi)^{2}},
\end{aligned}
$$


where $\epsilon^{ \pm} \equiv \frac{1}{2}\left(\epsilon_{x} \pm i \epsilon_{y}\right)$. The dominant contribution arising from the soft component of the deuteron occurs when $\alpha_{c} \sim \frac{1}{2}$, thus we may substitute $\alpha_{c}=\frac{1}{2}$. After factoring out the electromagnetic term, we identify the remaining integral in Eq. (3) [up to a scaling factor $f\left(l^{2} / s\right)$ ] as a particular contribution to the quark interchange mechanism (QIM) for the wide angle $p n$ scattering amplitude, $A_{p n}^{\mathrm{QIM}}\left(s, l^{2}\right)$. Then, summing over the struck quark contributions from photon scattering off neutron and proton [17], one obtains

$$
\begin{aligned}
T \approx & \frac{i\left(e_{u}+e_{d}\right)\left(\epsilon^{+}+\epsilon^{-}\right)}{2 \sqrt{s^{\prime}}} \\
& \times \int f\left(\frac{l^{2}}{s}\right) A_{p n}^{\mathrm{QIM}}\left(s, l^{2}\right) \Psi_{d}\left(\frac{1}{2}, p_{\perp}\right) \frac{d^{2} p_{\perp}}{(2 \pi)^{2}},
\end{aligned}
$$

where $e_{u}$ and $e_{d}$ are the electric charges of $u$ and $d$ quarks.

The factor $f\left(l^{2} / s\right)$ accounts for the difference between the hard propagators in our process and those occurring in wide angle $p n$ scattering. Within the Feynman mechanism, the interacting quark carries the whole momentum of the nucleon $\left(x_{1} \rightarrow 1\right)$, thus $f\left(l^{2} / s\right)=1$. Within the minimal Fock state approximation, the evaluation of the exact form of $f\left(l^{2} / s\right)$ requires calculating the sum of $\sim 10^{6}$ Feynman diagrams in which five hard-gluon exchanges are distributed between six quark lines. Here we use a qualitative evaluation of $f\left(l^{2} / s\right)$. The conditions $\alpha_{c} \sim \frac{1}{2}, 0<x_{1}<$ 1 according to Eq. (2) require $k_{1 \perp}^{2} \sim \frac{x_{1}\left(1-x_{1}\right) s^{\prime}}{2} \sim s^{\prime}$. Thus the dominant contribution in Eq. (4) is given by diagrams in which the struck quark exchanges a hard gluon with other quarks prior to the interaction with the photon (initial state short range $q q$ correlations). For these diagrams, the interchange of quarks between nucleons in both $\gamma d p n$ and $p n$ amplitudes is characterized by the same virtuality in the propagator of gluon exchange between nucleons, estimated at $\theta_{\text {c.m. }}=90^{\circ}$ to be $\sim\left(p_{t} / 3\right)^{2}$. All other propagators that define the short range part of the nucleon wave functions are the same. The other QIM diagrams with final-state $q q$ correlations only (which are suppressed in $\gamma d p n$ amplitude) enter in the $p n$ amplitude with larger virtuality for exchanged gluon between nucleons $\sim p_{t}^{2}$ and will be numerically small compared to the diagrams with initial state correlations. Thus no additional combinatorial factors enter in the hard scattering mechanism. Since the additional factor entering from the electromagnetic vertices is $\sim 1$, the function $f\left(l^{2} / s\right) \approx 1$ at $90^{\circ}$.

The amplitude (4) depends on only small relative momenta of the target nucleons, therefore we should use a standard nonrelativistic (NR) wave function; according to [14], $\Psi_{d}\left(\alpha, p_{\perp}\right)=(2 \pi)^{3 / 2} \Psi_{d}^{\mathrm{NR}}\left(p_{z}, p_{\perp}\right) \sqrt{m_{N}}$. We compute the differential cross section averaging $|T|^{2}$ over the spins of initial photon and deuteron and summing over the spins of the final nucleons. One obtains

$$
\begin{aligned}
\frac{d \sigma^{\gamma d \rightarrow p n}}{d t}= & \frac{1}{16 \pi} \frac{1}{\left(s-M_{d}^{2}\right)^{2}}|\bar{T}|^{2}=\frac{\alpha}{4} \frac{\pi^{3}}{9 s^{\prime 3}} \\
& \times \mid \int \sqrt{m_{N}} f\left(\frac{l^{2}}{s}\right) A_{p n}^{\mathrm{QIM}}\left(s, l^{2}\right) \\
& \times\left.\Psi_{d}^{\mathrm{NR}}\left(p_{z}=0, p_{\perp}\right) \frac{d^{2} p_{\perp}}{(2 \pi)^{2}}\right|^{2} .
\end{aligned}
$$

$A_{p n}^{\mathrm{QIM}}$ represents a sum of all leading-quark interchange diagrams with rather general structure of spectator quarkgluon systems. Thus $A_{p n}^{\mathrm{QIM}}$ absorbs the nonperturbative (noncalculable) part of our calculation. To proceed we observe that the quark interchange topologies shown to be the dominant contribution for fixed $\theta_{\text {c.m. }}=90^{\circ}$ high momentum transfer (nonstrange) baryon-baryon and mesonbaryon scattering [18]. Thus in the region of $\theta_{\text {c.m. }} \approx 90^{\circ}$ we replace $A_{p n}^{\mathrm{QIM}}$ by the experimental data, $A_{p n}^{\mathrm{Exp}}$. We also observe that the integrand in Eq. (5) is dominated by small values of $p_{\perp} \ll p_{A \perp}, p_{B \perp}$. Thus we evaluate $A_{p n}^{\mathrm{QIM}}$ at $t_{N}=\left(p_{B}-p_{d} / 2\right)^{2}$ by pulling this term out of the integral and expressing it through the differential cross section of $p n \rightarrow p n$ scattering, $\frac{d \sigma^{p n \rightarrow p n}}{d t}$ :

$$
\begin{aligned}
\frac{d \sigma^{\gamma d \rightarrow p n}}{d t}= & \frac{4 \alpha}{9} \pi^{4} \frac{1}{s^{\prime}} C\left(\frac{t_{N}}{s}\right) \frac{d \sigma^{p n \rightarrow p n}\left(s, t_{N}\right)}{d t} \\
& \times\left|\int \Psi_{d}^{\mathrm{NR}}\left(p_{z}=0, p_{\perp}\right) \sqrt{m_{N}} \frac{d^{2} p_{\perp}}{(2 \pi)^{2}}\right|^{2} .
\end{aligned}
$$

Equation (6) shows that the $\frac{d \sigma^{\gamma d \rightarrow p n}}{d t}$ depends on the soft component of the deuteron wave function, the measured high-momentum transfer $p n \rightarrow p n$ cross section, the scaling factor $C\left(\frac{t_{N}}{s}\right) \approx f^{2}\left(t_{N} / s\right) \approx 1$ at $\theta_{\text {c.m. }} \sim 90^{\circ}$ (and slowly varying as a function of $\theta_{\text {c.m. }}$ ), and the additional factor coming from the $\gamma-q$ interaction. Note that Eq. (6) is qualitatively different from the Glauber approximation. The latter is applicable only when intermediate nucleons are near on-mass shell. On the contrary Eq. (6) is derived when the mass ${ }^{2}$ of the intermediate state is $\left(\sim s \gg m_{N}^{2}\right)$. Our approach is close to that of Ref. [19] in which a nuclear amplitude is expressed as a product of a reduced nuclear amplitude and nucleon form factors. Here the nuclear amplitude is expressed in terms of the $p n$ hard scattering amplitude, and this allows us to calculate the absolute value of the cross section. Note that the $p n$ cross section scales as $s^{-10}$. This causes Eq. (6) to yield the same asymptotic $s^{-11}$ energy dependence (at fixed $t / s$ ) as provided by the quark counting rules.

In the numerical calculations we take $C\left(\frac{t_{N}}{s}\right)=1$. Our calculations are implemented using the Paris potential model of $\Psi_{d}^{\mathrm{NR}}$ [20] (but any realistic wave function would give the same result) and the experimental data from $[21,22]$ for $\frac{d \sigma^{p n \rightarrow p n}}{d t}$. The $p n \rightarrow p n$ data are not measured at the $t_{N}$ needed to evaluate Eq. (6), so an extrapolation is necessary. We determine an upper and a lower limit for $\frac{d \sigma^{p n-p n}}{d t}$ at $t_{N}$ using the existing $p n$ data at 


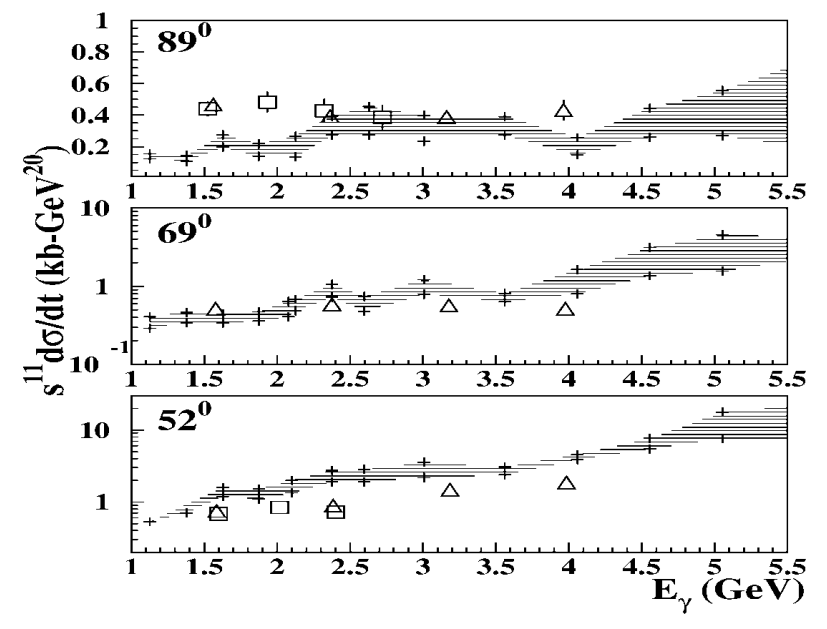

FIG. 2. The scaled differential cross sections as a function of the photon energy, for different values of the c.m. angle. Data are from [1] (triangles) and [3] (squares).

$t_{N}^{\min , \max }$ such that $-t_{N}^{\min }<-t_{N}<-t_{N}^{\max }$. Then Eq. (6) is computed using both the data at $t_{N}^{\min }$ and at $t_{N}^{\max }$, so that the calculation will produce a band. Figure 2 shows that calculations are in agreement with the measured differential cross sections. This agreement improves for larger $\theta_{\text {c.m. }}$ which confirms our expectation that $C\left(t_{N} / s\right) \approx 1$ at $\theta_{\text {c.m. }}=90^{\circ}$. The agreement with the data verifies our underlying hypothesis that the size of the photoproduction reaction is determined by the physics of high-momentum transfer contained in the hard scattering $N N$ amplitude. The short-distance aspects of the deuteron wave function are not important. This hypothesis, if confirmed by additional studies, may suggest the existence of a new type of quark-hadron "duality," where the sum of the "infinite" number of quark interactions could be replaced by the hard amplitude of $N N$ interaction.

It is worth noting that the deviations from the calculation based on the approximation $C\left(\theta_{\text {c.m. }}\right)=1$ seem to be consistent with $C$ being a function of $\theta_{\text {c.m. }}$ only. In particular, the whole set of available data can be described by taking $C\left(t_{N} / s\right)=\frac{-2 t_{N} / s^{\prime}}{1+2 t_{N} / s^{\prime}} \approx \frac{-t / s^{\prime}}{1+t / s^{\prime}}$ including even the data at $\theta_{\text {c.m. }}=36^{\circ}$, where $-t \leq 2 \mathrm{GeV}^{2}$ and the hard interaction mechanism cannot be applied. This may indicate that the connection between $N N$ and $\gamma d \rightarrow p n$ dynamics extends to a transitional region of $t$.

Although Eq. (6) is obtained utilizing only the quark interchange mechanism, the agreement of Fig. 2 may suggest that the result is more general-any hard interaction mechanism for $\gamma d \rightarrow h_{1}+h_{2}$ reaction could be expressed through the contribution of $p n \rightarrow h_{1} h_{2}$ hard scattering amplitude. More detailed data for angular distributions and final states involving different polarizations and compositions of final hadrons could provide an entirely new tool for the investigation of QCD dynamics of high energy nuclear reactions.

We thank S. Brodsky, A. Dieperink, and S. Nagorny for useful discussions and R. Holt and H. Gao for providing and explaining the experimental data. This work was supported in part by the U.S. Department of Energy and by the Academy of Science of Israel.

*Also at Yerevan Physics Institute, Yerevan, Armenia.

[1] C. Bochna et al., Phys. Rev. Lett. 81, 4576 (1998).

[2] J. Napolitano et al., Phys. Rev. Lett. 61, 2530 (1988); S. J. Freedman et al., Phys. Rev. C 48, 1864 (1993).

[3] J. E. Belz et al., Phys. Rev. Lett. 74, 646 (1995).

[4] S. J. Brodsky and B. T. Chertok, Phys. Rev. Lett. 37, 269 (1976).

[5] R. J. Holt, Phys. Rev. C 41, 2400 (1990).

[6] S. J. Brodsky and G. R. Farrar, Phys. Rev. Lett. 31, 1153 (1973); Phys. Rev. D 11, 1309 (1975); V. Matveev, R. M. Muradyan, and A. N. Tavkhelidze, Lett. Nuovo Cimento 7, 719 (1973).

[7] N. Isgur and C. H. Llewellyn Smith, Phys. Rev. Lett. 52, 1080 (1984); Phys. Lett. B 217, 535 (1989).

[8] A. Radyushkin, Acta Phys. Pol. B 15, 403 (1984).

[9] R. Feynman, Photon Hadron Interactions (W.A. Benjamin Inc., New York, 1972).

[10] A. E. Dieperink and S. I. Nagorny, Phys. Lett. B 456, 9 (1999).

[11] Yu. L. Dokshitzer, V. A. Khoze, A. H. Mueller, and S. I. Troyan, Basics of Perturbative QCD (Editions Frontieres, Paris, 1991).

[12] J. F. Gunion, S. J. Brodsky, and R. Blankenbecler, Phys. Rev. D 8, 287 (1973).

[13] G. P. Lepage and S. J. Brodsky, Phys. Rev. D 22, 2157 (1980).

[14] L. L. Frankfurt and M. I. Strikman, Phys. Rep. 76, 214 (1981); 160, 235 (1988).

[15] The light-cone four momentum is defined as $p=\left(p_{+}, p_{-}, p_{\perp}\right)$, where $p_{ \pm}=E \pm p_{z}$ and $\hat{z} \uparrow \downarrow \vec{q}$.

[16] We neglect the contribution of contact terms in the quark propagators, since they contribute only through the hard component of the deuteron wave function.

[17] We applied SU(6) symmetry for $u$ and $d$ quarks to sum diagrams. The introduced error is relatively small because the influence of SU(6) violation on the photon-quark interaction has opposing effects for the proton and neutron.

[18] C. White et al., Phys. Rev. D 49, 58 (1994).

[19] S. J. Brodsky and J. R. Hiller, Phys. Rev. C 28, 475 (1983).

[20] M. Lacombe et al., Phys. Lett. 101B, 139 (1981).

[21] M. L. Perl et al., Phys. Rev. D 1, 1857 (1970).

[22] J. L. Stone et al., Nucl. Phys. B143, 1 (1978). 